\title{
Angiopoietin-like protein 3, an emerging cardiometabolic therapy target with systemic and cell-autonomous functions
}

\section{Ruhanen, Hanna}

2020-12

Ruhanen , H , Haridas , P A N , Jauhiainen, M \& Olkkonen , V M 2020 , ' Angiopoietin-like protein 3 , an emerging cardiometabolic therapy target with systemic and cell-autonomous functions ' , Biochimica and Biophysica Acta. Molecular and Cell Biology of Lipids, vol. 1865 , no. 12 , 158791 . https://doi.org/10.1016/j.bbalip.2020.158791

http://hdl.handle.net/10138/333374

https://doi.org/10.1016/j.bbalip.2020.158791

cc_by_nc_nd

acceptedVersion

Downloaded from Helda, University of Helsinki institutional repository.

This is an electronic reprint of the original article.

This reprint may differ from the original in pagination and typographic detail.

Please cite the original version. 


\section{Angiopoietin-like protein 3, an emerging cardiometabolic therapy target with systemic and cell-autonomous functions}

Hanna Ruhanen ${ }^{1,2}$, P.A. Nidhina Haridas ${ }^{1}$, Matti Jauhiainen ${ }^{1}$, and Vesa. M. Olkkonen ${ }^{1,3^{*}}$

${ }^{1}$ Minerva Foundation Institute for Medical Research, Helsinki, Finland

${ }^{2}$ Molecular and Integrative Biosciences, University of Helsinki, Finland

${ }^{3}$ Department of Anatomy, Faculty of Medicine, University of Helsinki, Finland

${ }^{*}$ Corresponding author at: Minerva Foundation Institute for Medical Research, Biomedicum 2U, Tukholmankatu 8, Fl-00290 Helsinki, Finland; Tel +358-50-4112297, e-mail vesa.olkkonen@helsinki.fi 


\section{ABSTRACT}

Angiopoietin like protein 3 (ANGPTL3) is best known for its function as an inhibitor of lipoprotein and endothelial lipases. Due to the capacity of genetic or pharmacologic ANGPTL3 suppression to markedly reduce circulating lipoproteins, and the documented cardioprotection upon such suppression, ANGPTL3 has become an emerging therapy target for which both antibody and antisense oligonucleotide (ASO) therapeutics are being clinically tested. While the antibody is relatively selective for circulating ANGPTL3, the ASO also depletes the intra-hepatocellular protein, and there is emerging evidence for cellautonomous functions of ANGPTL3 in the liver. These include regulation of hepatocyte glucose and fatty acid uptake, insulin sensitivity, LDL/VLDL remnant uptake, VLDL assembly/secretion, polyunsaturated fatty acid (PUFA) and PUFA-derived lipid mediator content, and gene expression. In this review we elaborate on (i) why ANGPTL3 is considered one of the most promising new cardiometabolic therapy targets, and (ii) the present evidences for its intra-hepatocellular or cell-autonomous functions.

Keywords: Angiopoietin-like 3, lipoprotein lipase, lipid metabolism, intracellular function, hepatic lipids, therapy target 


\section{Introduction}

Angiopoietin like 3 (ANGPTL3) belongs to the angiopoietin-like protein family consisting of 8 members structurally similar to angiopoietins [1]. ANGPTL3 was first identified by Conklin et al. [2]. It is mainly expressed in liver; very low expression is also detectable in kidney podocytes but its role in podocyte function is not fully understood. The protein consists of an $\mathrm{N}$-terminal signal peptide, a coiled-coil region, a linker region, and a C-terminal fibrinogenlike domain (FLD; Fig. 1). ANGPTL3 expression is regulated positively by liver $X$ receptor (LXR) and negatively by insulin, leptin and thyroid hormone [3-6]. The main function of ANGPTL3 is in the regulation of lipid metabolism, where it acts synergistically with the related ANGPTL4 and ANGPTL8, forming a functionally essential protein-protein complex with the latter [7,8]. Unlike ANGPTL3, ANGPTL4 and ANGPTL8 are expressed in both adipose tissue and liver $[6,9,10]$.

Angpt/3 was in an early study found to associate with a hypolipidemia trait in a moderately obese mouse strain [11], and knock-out of Angpt/3 was reported to result in a hypotriglyceridemia phenotype [12]. Later, genome-wide association study (GWAS) in human subjects revealed that markers in the region of ANGPTL3 are associated with circulating triglyceride (TG) levels [13]. Moreover, loss-of-function (LOF) variants of ANGPTL3 were found to associate with reduced plasma LDL- and HDL-cholesterol (LDL-C, HDL-C) and TG [14]; Several other studies soon replicated these observations $[10,15,16]$. The major known function of ANGPTL3 is to inhibit the activity of lipoprotein lipase (LPL), an enzyme that hydrolyses TG in TG-rich lipoproteins (TRL) [10,17]. Moreover, ANGPTL3 also inhibits endothelial lipase (EL) [18], which provided a putative explanation for its effects on circulating HDL-C as shown in vivo in mouse [19]. However, ANGPTL3 deficient human subjects displayed similar EL activity as in control individuals, suggesting that other mechanisms may account for the reduction of HDL levels in these subjects [16]. Importantly, ANGPTL3 LOF variant carriers are protected from coronary artery disease [20]. Due to this cardioprotective effect, ANGPTL3 has emerged as a novel therapeutic target for cardiovascular diseases (CVD). ASO and monoclonal antibody-based therapeutics targeting ANGPTL3 are in clinical trials [21-25]. While the function of ANGPTL3 in regulating plasma lipoprotein levels is rather well studied and reviewed [1,26-29], very little is known about the putative intracellular functions of ANGPTL3. Since ANGPTL3-targeting ASO drugs, which inhibit the hepatocellular expression of the protein, are under clinical trial, it is important to also understand the intracellular functions of ANGPTL3. In this review we 
address two crucial aspects of the protein: (i) Why is ANGPTL3 considered a promising therapeutic target for CVD? (ii) What is the evidence for intra-hepatocellular or cellautonomous functions of ANGPTL3, and what are the implications of such functions?

\section{Why is ANGPTL3 a promising cardiovascular therapy target?}

Due to its role as a crucial physiologic regulator of LPL and EL activity, ANGPTL3 has a major impact on the circulating lipoproteins, the loss of its function resulting in marked reductions of all major lipoprotein classes. Moreover, evidence from pre-clinical studies suggests that inactivation of ANGPTL3 reduces VLDL-TG secretion [19,23,30,31] and enhances the hepatic uptake of apoB100-containing lipoproteins [32] as well as VLDL-TG uptake into oxidative tissues [33]. The role of ANGPTL3 in human lipid metabolism was revealed in 2010 through the identification of individuals carrying LOF mutations in ANGPTL3 [14]. Genetic deficiency of ANGPTL3 was found to cause familial combined hypolipidemia (FHBL2, OMIM \#605019) characterized by very low plasma TG, LDL-C and HDL-C concentrations. A number of LOF mutations have been characterized in the gene [14,34-36]. Of these, ANGPTL3S17X is particularly interesting since it completely eliminates the protein and has been detected in three generations in the town of Campodimele in Italy $[36,37]$. The life expectancy of both females and males in this small town is exceptionally high, approximately $95 \mathrm{y}$. The inbred inheritance of ANGPTL3 LOF mutations is considered one of the factors underlying this remarkable longevity.

A crucial question has arisen whether genetic abrogation of ANGPTL3 associated with reduced circulating lipoprotein concentrations is protective against CVD. An extensive meta-analysis (a total of 21,980 CVD patients and 158,200 controls) demonstrated that carriers of ANGPTL3 LOF mutations have $34 \%$ lower odds of CVD than control subjects [20]. Individuals belonging to the lowest tertile of circulating ANGPTL3 concentration presented with a $29 \%$ lower myocardial infarction (MI) risk than the highest tertile. A reduction of CVD risk among ANGPTL3 LOF mutation carriers was replicated by Dewey et al. [21], who sequenced ANGPTL3 exons in 58,335 participants of the DiscovEHR genetics study. Here, subjects heterozygous for ANGPTL3 LOF variants displayed significantly lower serum TG $(-27 \%)$, HDL-C (-4\%) and LDL-C (-9\%) concentrations than participants lacking the variants. Moreover, analysis of ANGPTL3 LOF variants in 13,102 coronary artery disease (CAD) patients and 40,430 controls showed that the LOF variants 
were found in $0.33 \%$ of the cases and in $0.45 \%$ of the controls (adjusted odds ratio, 0.59 ; $95 \% \mathrm{Cl}, 0.41$ to $0.85 ; \mathrm{p}=0.004)$. These intriguing observations were further confirmed by follow-up studies employing four large population cohorts. An inverse-variance-weighted fixed-effects meta-analysis combining the DiscovEHR and the other population studies suggested an odds ratio of $0.61(95 \% \mathrm{Cl}, 0.45$ to $0.81 ; \mathrm{p}<0.001)$ [21]. Interestingly, Lotta et al. [38] reported in a large human material (392,220 participants) that ANGPTL3 LOF variants were associated with greater protection against coronary disease than other LDLC-lowering genetic mechanisms (ANGPTL3 loss-of-function variants: odds ratio, 0.66; 95\% $\mathrm{Cl}, 0.52-0.83$; 58 other LDL-C-lowering variants: odds ratio, $0.90 ; 95 \% \mathrm{Cl}, 0.89-0.91$; $\mathrm{P}$ for heterogeneity $=0.009$ ).

The above epidemiological studies suggested that the plasma lipid/lipoprotein lowering observed in ANGPTL3 LOF carriers (the strongest effect being detected on TG levels) may precipitate as a drop of cardiovascular risk. The anticipated adverse effects of HDL lowering were apparently overridden by the beneficial reduction of TG and LDL-C. Reduction or inactivation of ANGPTL3 was, based on the above observations, considered a promising candidate CVD therapeutic strategy, and is being actively pursued.

What have we learned from the clinical trials on ANGPTL3 targeting? Trials on the suppression or inhibition of ANGPTL3 are ongoing at phases 2 and 3. The therapeutic agents under study are antisense oligonucleotides (AKCEA/IONIS-ANGPTL3-LRx, a GalNac-conjugated ASO, presently called Vupanorsen), which suppress the hepatic expression of $A N G P T L 3$, or a human monoclonal antibody that inactivates circulating but not intra-hepatic ANGPTL3 (Evinacumab, Regeneron Pharmaceuticals). Moreover, in vivo genome editing by CRISPR-Cas9 technology is being developed as a tool to introduce LOF mutations in ANGPTL3. This novel approach has yielded the first promising results in a mouse model [39]. Results from both the ASO and the antibody trials have revealed significant decreases of plasma ANGPTL3 levels coinciding with reductions of all major lipoprotein classes. Graham et al. [23] showed in a phase 1 clinical trial that, after 6 weeks of ASO treatment on healthy adults, subjects receiving single or multiple doses of the ASO displayed 46.6 to $84.5 \%$ reductions of plasma ANGPTL3 protein $(P<0.01$ for all doses vs. placebo) and marked drops in the levels of TG (reductions of 33.2 to $63.1 \%$ ), LDL-C (1.3 to $32.9 \%$ ), VLDL-C (27.9 to $60.0 \%$ ), non-HDL-C (10.0 to $36.6 \%$ ), apoB-100 (3.4 to $25.7 \%$ ), and apoC-III (18.9 to $58.8 \%$ ). Of note, although thrombocytopenia has been reported as an 
adverse side effect of certain ASO therapies [40,41], this or other serious treatmentemergent adverse events (TEAE) or discontinuation of the therapy were not documented during the trial.

After successful lipid lowering in mice and cynomolgus monkeys [19,21], the monoclonal antibody Evinacumab was administered in 83 healthy human volunteers with mildly to moderately elevated TG $(150-450 \mathrm{mg} / \mathrm{dl})$ or LDL-C $(100 \mathrm{mg} / \mathrm{dl})$ by Dewey et al. [21]. This resulted in a dose-dependent reduction in fasting TG of up to $76 \%$ and LDL-C of up to $23 \%$. Similar observations were recently reported from a clinical trial on hypertriglyceridemic subjects by Ahmad et al. [24], who also measured significant reductions of VLDL-C in the Evinacumab-treated groups, in the absence of serious TEAE or discontinuation of the therapy.

When one considers subjects with familial hypercholesterolemia $(\mathrm{FH})$, for whom the efficacy of statin and PCSK9 inhibitor therapies is often not sufficient to reach their target lipid levels, ANGPTL3 targeting might give additional benefit. Consistent with this idea, Evinacumab administered for 4 weeks on top of intense lipid-lowering therapy resulted in a further reduction of TG (by 47\%), LDL-C (by 50\%), and HDL-C (by 36\%) in a group of 9 homozygous FH patients [22]. A more extensive phase 3 trial (ELIPSE HoFH; NCT03399786) on homozygous $\mathrm{FH}$ patients is ongoing with promising $1^{\text {st }}$ endpoint results $(49 \%$ reduction of LDL-C; significant drop of TG, TC, non-HDL-C and apoB). The idea of combining Evinacumab with other lipid-lowering therapies to reach maximal efficiency was recently tested in a pre-clinical setting by Pouwer et al. [42]. The authors studied the effect of 'triple therapy' consisting of evinacumab, the PCSK9 inhibitor alirocumab, and atorvastatin, on atherosclerosis in APOE ${ }^{*} 3$-Leiden.CETP mice, a well-established model mimicking human hyperlipidemia. After 13-week western-type diet the mice received the pharmaceuticals for 25 weeks, the 'triple therapy' yielding clearly better results than atorvastatin alone or atorvastatin + alirocumab ('double treatment'). Atorvastatin alone reduced lesion progression by $28 \%$, the double treatment completely blocked lesion progression and diminished lesion severity, increasing their collagen content, while the triple therapy regressed the lesions in thoracic aorta by $50 \%$ and in the aortic root by $36 \%$, increasing their collagen and reducing their macrophage content. All the above pre-clinical and clinical trials strongly suggest that residual risk remaining after statin-PCSK9 inhibitor treatment can be further diminished via blocking ANGPTL3 function. It is noteworthy that both the ASO and 
antibody therapies elicited apoB reductions ranging from $-14 \%$ to $-46 \%$. Considering a recent report suggesting that the clinical benefit of TG and LDL-C lowering may in fact be proportional to the absolute change in apoB [43], this may be a crucial effect of ANGPTL3targeting therapy. The above studies and the evidence in favor of employing ANGPTL3 as cardiometabolic therapy target are summarized in Table 1.

Table 1. Evidence for lipid lowering and cardioprotection upon loss or suppression of ANGPTL3 activity/expression.

\begin{tabular}{|c|c|c|c|}
\hline Method & Species & Documented effect & Reference(s) \\
\hline \multirow[t]{7}{*}{$\begin{array}{l}\text { Genetic loss-of- } \\
\text { function (LOF) }\end{array}$} & Human & $\begin{array}{l}\text { Reduction of TG, LDL-C, HDL- } \\
\text { C }\end{array}$ & {$[14]$} \\
\hline & Human & $\begin{array}{l}\text { Reduction of TG, TC, LDL-C, } \\
\text { HDL-C, ApoB, ApoA-I }\end{array}$ & {$[15,44]$} \\
\hline & Human & $\begin{array}{l}\text { LOF carriers, OR }{ }^{1} \text { of CAD } 0.66 \text {; } \\
\text { Lowest tertile of ANGPTL3 } \\
\text { conc. OR } 0.65 \text { relat. to highest } \\
\text { tertile; Reduction of TG, TC, } \\
\text { LDL-C HDL-C }\end{array}$ & [20] \\
\hline & Human & $\begin{array}{l}\text { LOF carriers, OR of CAD 0.59; } \\
\text { Reduction of TG, TC², LDL-C, } \\
\text { HDL-C }\end{array}$ & [21] \\
\hline & Human & $\begin{array}{l}\text { LOF carriers, OR of CHD 0.66; } \\
\text { Other LDL-C lowering variants, } \\
\text { OR } 0.90\end{array}$ & [38] \\
\hline & Human & $\begin{array}{l}\text { Reduction of TG, TC, LDL-C, } \\
\text { HDL-C, ApoB, ApoA-I, FFA; } \\
\text { Increase of IS }{ }^{3}\end{array}$ & [16] \\
\hline & Mouse, Angpt/3\%- & $\begin{array}{l}\text { Reduction of TG, TC; Increase } \\
\text { of IS and WAT }{ }^{4} \text { glucose uptake }\end{array}$ & {$[12,33]$} \\
\hline \multirow[t]{8}{*}{$\begin{array}{l}\text { Antibody } \\
\text { inhibition }\end{array}$} & Human & $\begin{array}{l}\text { Reduction of TG }(\max -76 \%)^{5} \text {, } \\
\text { LDL-C }(-23 \%) \text {, HDL-C }(-18 \%)\end{array}$ & [21] NCT01749878 \\
\hline & Human $\left(\mathrm{FH}^{6}\right)$ & $\begin{array}{l}\text { Reduction of LDL-C (mean } \\
-49 \%) \text {, apoB }(-46 \%) \text {, non-HDL- } \\
\text { C }(-49 \%) \text {, TG }(-47 \%) \text {, HDL-C } \\
(-36 \%)\end{array}$ & [22] NCT02265952 \\
\hline & Human (FH) & $\begin{array}{l}\text { Reduction of LDL-C (-49\%), } \\
\text { TG, TC, non-HDL-C and apoB }\end{array}$ & NCT03399786 \\
\hline & Human (hyper-TG) & $\begin{array}{l}\text { Reduction of TG (max }-88 \%) \text {, } \\
\text { TC }(-34 \%) \text {, LDL-C }(-25 \%) \text {, } \\
\text { non-HDL-C }(-40 \%) \text {, VLDL-C } \\
(-91 \%) \text {, apoB }(-31 \%), \text { HDL-C } \\
(-28 \%)\end{array}$ & $\begin{array}{r}\text { [24] NCT01749878 } \\
\text { NCT02107872 }\end{array}$ \\
\hline & Cynomolgus monkey & $\begin{array}{l}\text { Reduction of TG, non-HDL-C, } \\
\text { HDL-C }\end{array}$ & [19] \\
\hline & $\begin{array}{l}\text { Mouse, } \\
\text { APOE*}^{3} 3 \text { Leiden.CETP }\end{array}$ & $\begin{array}{l}\text { Lesion area reduction } 39 \% \\
\text { Necrotic area reduction } 45 \% \\
\text { Reduction of TG, TC. VLDL-C }\end{array}$ & [21] \\
\hline & 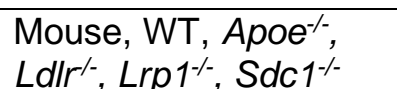 & $\begin{array}{l}\text { Reduction of TG, TC, LDL-C, } \\
\text { VLDL-C }\end{array}$ & [31] \\
\hline & $\begin{array}{l}\text { Mouse, WT, } \\
\text { dyslipidemic }\end{array}$ & $\begin{array}{l}\text { Reduction of TG, TC, LDL-C, } \\
\text { HDL-C }\end{array}$ & [19] \\
\hline
\end{tabular}




\begin{tabular}{|c|c|c|c|}
\hline & $\begin{array}{l}\text { Mouse, } \\
\text { APOE*3Leiden.CETP }\end{array}$ & $\begin{array}{l}\text { Evinacumab + alirocumab + } \\
\text { atorvastatin, lesion regression; } \\
\text { Reduction of TC, non-HDL-C, } \\
\text { VLDL-C, LDL-C }\end{array}$ & [42] \\
\hline ASO & Human & $\begin{array}{l}\text { Reduction of TG (max }-63 \%), \\
\text { LDL-C (-33\%), VLDL-C } \\
(-60 \%) \text {, non-HDL-C (-37\%), } \\
\text { ApoB (-26\%), ApoC-III (-59\%), } \\
\text { ApoA-I, Lp(a) }\end{array}$ & [23] NCT02709850 \\
\hline & Mouse, Ldlr'- & $\begin{array}{l}\text { Lesion area reduction } 52 \% \\
\text { Reduction of TG, LDL-C }\end{array}$ & [23] \\
\hline siRNA & $\begin{array}{l}\text { Mouse, WT, ob/ob } \\
\text { hCETP/ApoB-100 } \\
\text { Apobec }{ }^{-/} \text {hApoB Tg } \\
\text { Ldlr }^{\prime-}\end{array}$ & $\begin{array}{l}\text { Reduction of TG, HDL-C, LDL- } \\
\text { C } \\
\text { Reduction of of TG, LDL-C } \\
\text { Minimal effect on LDL-C }\end{array}$ & [32] \\
\hline $\begin{array}{l}\text { CRISPR-Cas9 } \\
\text { base editing }\end{array}$ & WT, Ldl/r'- & Reduction of TG, TC & [39] \\
\hline
\end{tabular}

${ }^{1}$ Odds ratio; ${ }^{2}$ Total cholesterol; ${ }^{3}$ Insulin sensitivity; ${ }^{4}$ White adipose tissue, ${ }^{5}$ For human clinical studies a maximum or mean (for ref. [22]) \% reduction of plasma lipids or lipoproteins are given. The numbers are not directly comparable since they have been picked from different single or multiple dose regimes in the different studies. ${ }^{6}$ Familial hypercholesterolemia; ${ }^{7}$ Cholesterol ester transfer protein

To conclude, ANGPTL3 is currently considered a highly promising cardiovascular therapy target. Its inactivation alone or especially in combination with other lipid-lowering therapies holds great promise particularly for subjects with hard-to-treat dyslipidemias inflicting a high CVD risk. Moreover, additional benefit concerning metabolic disease is suggested by the observations demonstrating that ANGPTL3 deficiency is associated with increased insulin sensitivity in both human subjects [16] and mice [33]. Even though the Evinacumab antibody therapy has shown positive results, the ASO therapy and the emerging CRISPR-Cas9 approach are likely to gain ground in the future. However, both of these induce long-term or permanent suppression of the hepatic ANGPTL3 expression, thus also afflicting the putative intra-hepatocellular or cell-autonomous functions of ANGPTL3 - detailed study of these functions is therefore a necessity.

\section{ANGPTL3 and non-alcoholic fatty liver disease (NAFLD)}

\subsection{Familial hypobetalipoproteinemias and fatty liver}

As indicated above, LOF mutations in the ANGPTL3 gene cause familial combined hypolipidemia 2 (FHBL2, OMIM \#605019) associated with significantly reduced plasma levels of all apoB-100 containing lipoproteins as well as HDL. Also several variants of the 
$A P O B$ gene are connected to low plasma apoB-100 and LDL-C concentrations, causing familial hypobetalipoproteinemia 1 (FHBL1, OMIM \#615558). In FHBL1 apoB-100 plasma levels are generally $<5$ th percentile whereas LDL-C concentrations are in the range of 200$500 \mathrm{mg} / \mathrm{L}$ [45,46]. Of note, FHBL1 subjects are prone to develop NAFLD [47-49]. Using stable isotope techniques it was demonstrated that heterozygous FHBL1 subjects have a significantly reduced hepatic apoB-100 secretion rate, attenuated production of LDLassociated apoB-100, and highly enhanced catabolism of VLDL particles [50,51].

Although an elevated prevalence of liver steatosis in FHBL1 has been firmly documented, the hepatic neutral lipid levels in FHBL2 are as yet not well established. There are only a few investigations addressing the hepatic lipid status in ANGPTL3 deficient subjects. Musunuru et al. [14] characterized one ANGPTL3 LOF mutation kindred, and signs of fatty liver were not detected, suggesting that ultrasonography could be employed to discriminate ANGPTL3 (FHBL2) from $A P O B$ deficiency (FHBL1), but not from hypobetalipoproteinemia due to PCSK9 mutations, where advanced fatty liver is usually not observed. Minicocci et al. [44] studied 115 FHBL2 individuals with 13 different ANGPTL3 variants (14 homozygotes, 8 compound heterozygotes, and 93 heterozygotes) and about 400 control subjects. Detailed examination for the presence of hepatic steatosis was performed among 64 ANGPTL3 S17X variant carriers and 103 control subjects. The authors did not observe increased prevalence of liver steatosis in this FHBL2 group compared with the non-carrier controls. Furthermore, there was no association between the severity of steatosis and the FHBL2 status. Di Costanzo et al. [52] carried out a direct comparison of the hepatic lipid phenotypes of FHBL1 and FHBL2. Altogether 350 subjects, 67 heterozygous carriers of $A P O B$ mutations (FHBL1 subjects), 57 heterozygote and 6 homozygote ANGPTL3 variant carriers (FHBL2 subjects), and 220 normolipidemic controls were included. Prevalence and degree of hepatic steatosis were analyzed by ultrasonography. The major outcome of this study was that, when comparing to the controls, the prevalence and severity of hepatic steatosis were significantly increased in heterozygous FHBL1 subjects $(P<0.001)$, while the FHBL2 participants did not differ from controls. The hepatic consequences of FHBL2 thus seem to be strikingly different from those of $A P O B$ linked FHBL1 in which elevated prevalence of hepatic steatosis has been consistently demonstrated.

3.2 Plasma ANGPTL3 concentration, insulin resistance and liver disease 
Experimental studies have suggested a potential role of the angiopoietin-like proteins, especially ANGPTL3, in the pathogenesis of metabolic syndrome, hepatic steatosis, and more advanced forms of liver disease. One study focused on measuring ANGPTL3 plasma levels in patients with definite nonalcoholic steatohepatitis ( $N A S H, n=40$ ), borderline $\mathrm{NASH}$ $(n=8)$, simple fatty liver $(n=9)$, and healthy control subjects without liver disease $(n=14)$. The results suggested that plasma ANGPTL3 levels are increased in the more severe cases of NAFLD in association with insulin resistance [53]. Another single center, cross-sectional study among approximately 1000 Japanese undergoing routine health checks also observed elevated circulating ANGPTL3 concentrations in subjects with impaired liver function and hepatic inflammation [54]. The DiOGenes (Diet, Obesity and Genes) study examined the role of ANGPTL3 in lipoprotein metabolism and liver health status [55]. The authors measured ANGPTL3 levels in relation to body mass index, plasma lipid profile, and markers of hepatic steatosis before and during weight loss. Moreover, genetic variants affecting plasma ANGPTL3 levels were defined through protein quantitative trait locus ( $\mathrm{PQTL}$ ) analysis. The major findings were a strong negative correlation between plasma ANGPTL3 concentration and ASAT activity, and a positive association with cytokeratin 18 (CK-18), independent of weight loss. CK-18 is a major hepatic intermediate filament protein, and plasma CK-18 acts as a biomarker for the apoptotic death of hepatocytes, NASH and hepatic inflammation [56]. The authors of the DiOGenes study suggested that elevation in plasma ANGPTL3 concentration results from hepatic inflammation, or that ANGPTL3 itself could play a role in the development of liver malfunction. In vitro support for these suggestions is provided in the study by Szalowska et al. [57], where inflammation was induced in vitro in human liver tissue, and ANGPTL3 appeared as a biomarker associated with the inflamed status. Of note, no increase of liver fat content was reported in mice treated with ANGPTL3 ASO, and no significant change in biomarkers of liver function was observed in human subjects who received the ASO [23], in contrast to the hepatotoxicity frequently observed upon treatments with inhibitors targeting the microsomal triglyceride transfer protein (Lomitapide; [58]) or apoB-100 (Mipomersen; [59]).

Interestingly, Ruhanen et al. [60] recently demonstrated that in human hepatocytes subjected to ANGPTL3 knock-down (KD) several lipid classes were enriched in $n-6$ and $n-3$ polyunsaturated fatty acids (PUFA), and this phenomenon coincided with an elevation of a number of PUFA-derived lipid mediators relevant for the resolution of inflammation and protection from hepatic insulin resistance and steatosis (see paragraph 
7). However, because of the controversy on the applicability of non-invasive biomarkers as metrics of liver derangements, future investigations should analyze liver biopsies to more reliably evaluate the role of ANGPTL3 in liver disease.

\section{Modulation of hepatic lipoprotein uptake by ANGPTL3}

The reduction of circulating lipoproteins upon ANGPTL3 LOF can be partially explained by the increase in LPL and EL activity caused by the loss of secreted ANGPTL3. However, the loss of ANGPTL3 also may contribute to this by enhancing hepatic lipoprotein uptake. The decrease in LDL-C upon ANGPTL3 KD in vivo in mouse appeared to be mediated via the LDL receptor (LDLR), as suggested by experiments employing Ldlr-deficient Apobec $1^{-1-} / A p o B-100$ transgenic mice [32]. However, contradicting in vivo results were reported by Graham et al. [23] who found a similar drop of LDL-C upon ANGPTL3 ASOtreatment in both wild-type and $L d l r^{-}$mice, implying that the drop of LDL in this case may not depend on functional LDLR. While the best characterized high affinity receptor for LDL is LDLR, a recent LDL transcytosis study by Armstrong et al. [61] showed that LDL colocalized partially with the scavenger receptor SR-BI and overexpression of SR-BI increased LDL transcytosis, whereas SR-BI knockdown by siRNA significantly inhibited this process. This suggests that hepatic SR-BI could facilitate LDL uptake in Ld/r' mice and thus contribute to the reduced plasma LDL-C levels upon suppression of ANGPTL3, an issue that warrants further study.

Interestingly, deficiency of ANGPTL3 in the HuH7 and HepG2 hepatocellular carcinoma cells in vitro was shown to result in an elevated rate of LDL and VLDL uptake. Further, the ANGPTL3 depletion induced intracellular accumulation of long-chain TG and apoB-100, as well as elevated expression of both LDLR and LDLR-related protein 1 (LRP1) [32] (Fig. 2). Since ANGPTL3 function through inhibition of LPL is hardly relevant in experiments employing cultured hepatocytes, the above in vitro results suggest that ANGPTL3 modulates LDL uptake through an intracellular, or cell-autonomous mode of action. The study of Wang et al. [31] employing antibody inhibition of ANGPTL3 in mouse did not observe LDLRdependency of the drop of circulating LDL, and this is no doubt the case also in homozygous human FH patients carrying $L D L R$ null mutations but still exhibiting a marked drop of LDL$C$ upon Evinacumab therapy [22]. These observations suggest that inhibition of the circulating ANGPTL3 by the monoclonal antibody, which most likely dampens the hepatic 
VLDL secretion through reduction of FA flux towards the liver, results in a marked drop of circulating LDL independent of the LDLR pathway (Fig. 2; see below).

\section{ANGPTL3 regulates hepatocyte VLDL secretion.}

Plasma VLDL and LDL levels are very low in ANGPTL3 deficient humans and mice. To what extent can this be attributed to inhibition of VLDL secretion by hepatocytes? Targeting ANGPTL3 with a monoclonal antibody (Evinacumab, REGN1500) in a number of genetically modified mouse models reduced the plasma total levels of TG and cholesterol, including those in the VLDL fraction, and the hepatic TG secretion, without effect on plasma apoB concentration or VLDL/LDL particle numbers [19,21,31]. The study of Wang et al. [31] suggested a selective effect of ANGPTL3 inhibition on hepatic TG but not apoB secretion. Such an effect could be explained by enhanced LPL activity in oxidative tissues and possibly reduced adipose tissue lipolysis [62], resulting in a reduced flux of FA to the liver and, as a consequence, reduced assembly and secretion of large VLDL particles (Fig. 2). A somewhat contradicting observation was made by $\mathrm{Xu}$ et al. [32], who reported reduced apoB-100 accumulation in the growth medium of $\mathrm{HuH} 7$ and HepG2 cells subjected to ANGPTL3 KD. This finding may also suggest that the consequences of in vivo inhibition of circulating ANGPTL3 may include cross-talk between liver and tissues such as WAT, a connection that is lacking when studying hepatocytes in culture. According to Wang et al. [31], the turnover of LDL or VLDL was not affected in mice treated with ANGPTL3-inhibiting antibody, consistent with the notion that the observed $\beta$-lipoprotein reduction was largely due to inhibition of VLDL secretion.

ASO-mediated silencing of Angpt/3 in human subjects and mouse liver affects, in addition to circulating ANGPTL3, the intra-hepatocellular protein. In both humans and mice, similar results of ASO treatment were reported: In humans, the treatment resulted in a significant reduction of TG, LDL-C, VLDL-C, non-HDL-C, apoB-100 and ApoC-III [23]. In the siRNA-mediated Angptl3 knock-down study of $X u$ et al. [32], lipid phenotypes resembling the above were observed in both wild-type and obese mice, while in more 'human-like' hCETP/ApoB-100 double transgenic animals, no drop of HDL-C was evident. Further, silencing of ANGPTL3 in HuH7 and HepG2 hepatocellular carcinoma cell lines caused a reduction in apoB-100 and TG secretion. Likewise, ANGPTL3 KD in non-cancerous 
immortalized human hepatocytes $(\mathrm{IHH})$ induced a shift from the secretion of large VLDL1like particles to more lipid-poor VLDL2-like particles upon insulin stimulation [30]. Taken together, these observations suggest that intracellular ANGPTL3 acts as a regulator of VLDL secretion. However, the underlying mechanism(s) remain poorly understood. In a recent report, Ruhanen et al. [60] showed that the cholesterol ester (CE) levels are reduced in ANGPTL3 depleted hepatocytes, apparently mediated by downregulation of acylCoA:cholesterol acyltransferase1 (SOAT1) expression. Burnett et al. [63] have previously demonstrated a role of SOAT1 in VLDL secretion from hepatocytes, and the cellular CE levels are reported to affect apoB-100 and VLDL secretion [64,65]. The defects observed in VLDL secretion upon ANGPTL3 depletion may thus, in part, depend on the availability of intra-hepatocellular CE.

Another clue to the mechanisms that could putatively explain the impact of ANGPTL3 on VLDL secretion is provided by the study of Ruhanen et al. [60], who showed that ANGPTL3 knock-down hepatocytes are enriched with polyunsaturated fatty acid (PUFA)-containing lipid species (see paragraph 7). The changes in the FA composition may affect the assembly, composition and secretion of VLDL particles and also their further hydrolysis properties by LPL. Importantly, n-3 and n-6 PUFA intake in humans has been shown to reduce plasma VLDL, mainly by enhancing VLDL lipolysis and uptake [66]. On the other hand, lysophosphatidylcholine acyltransferase 3 (LPCAT3) deficient hepatocytes depleted of arachidonic acid (20:4n-6) containing phospholipid species were unable to secrete mature VLDL particles, apparently due to defects in forming the membrane curvature required for VLDL assembly [67]. This illustrates well how a change in the FA composition of lipids can modulate VLDL secretion. More studies, however, are required to determine whether the PUFA enrichment in ANGPTL3-depleted hepatocytes indeed affects the secretion of VLDL. We are tempted to speculate that the accumulation of PUFAs might favor the production of small VLDL particles or ones acting as a better substrate for LPL and hepatocellular uptake.

\section{Connection of ANGPTL8 and ANGPTL3 function in the liver}

Human ANGPTL8, a functional partner of ANGPTL3, is predominantly expressed in the liver, while mouse Angptl8 is expressed in both the liver and the adipose tissues. Studies on ANGPTL8 have provided evidence for intracellular functions in the regulation of metabolism and inflammatory responses. ANGPTL8 was reported to alleviate insulin resistance in 
HepG2 cells via the Akt-GSK3ß or Akt-FOXO1 pathway [68]. Moreover, ANGPTL8 oligomers were demonstrated dampen $\mathrm{NF}-\mathrm{kB}$ activation through selective autophagic degradation of IKK in HepG2 cells, with consistent in vivo observations made in LPStreated mice [69]. Importantly, ANGPTL3 relies on ANGPTL8 in the regulation of LPLmediated TG hydrolysis: ANGPTL8 promotes the ability of ANGPTL3 to bind and inhibit LPL [70]. On the other hand, ANGPTL8 itself has a functional LPL-inhibitory motif, but inhibits LPL and increases plasma TG levels only in the presence of ANGPTL3 [71]. ANGPTL8 can form complexes with ANGPTL3 with varying stoichiometric ratios, the assembly of these complexes taking place intra-hepatocellularly. In this process ANGPTL8 binds to the Nterminal domain of ANGPTL3 and mediates proteolytic cleavage of ANGPTL3 via exposure of its cleavage site or recruitment of relevant hepatic proteases [7,72] (Fig. 3). Thus far there are no data available on a putative role of ANGPTL8 in hepatic lipid metabolism under conditions in which the liver is devoid of ANGPTL3 biosynthesis, i.e. FHBL2.

\section{Impacts of ANGPTL3 on the fatty acid (FA) composition of hepatocyte lipids and on PUFA-derived lipid mediators}

To date, only a handful of studies have addressed the putative effects of ANGPTL3 on intrahepatic lipid metabolism and in even fewer studies detailed information on intrahepatic lipid molecular species composition or FA profile has been reported. The limited information on how ANGPTL3 affects hepatic FA has been gained from animal or in vitro studies. In a study conducted using mice kept on a high-fat high-cholesterol diet, neutralizing ANGPTL3 in the circulation with a multiple dose treatment of the antibody Evinacumab (REGN1500) did not change the hepatic expression of genes related to de novo lipogenesis or FA oxidation, and, consistently, there was no change in liver fat content [19]. Similarly, there were no changes in the transcription of genes involved in fatty acid synthesis, desaturation, or $\beta$-oxidation in the livers of Angp/t3\% mice [33]. In these knock-out mice on a chow diet, the hepatic level of FA 18:2n-6 (linoleic acid) or 16:1n-7 (palmitoleic acid) did not change nor did the ratio of the exogenous FAs 18:2n-6 or 18:3n-3 ( $\alpha$-linolenic acid) to 16:1n-7.

In cultured human hepatocytes subjected to ANGPTL3 knock-down (KD), however, changes in lipid metabolism and in the FA composition of lipids have been observed. Both transcriptomics and lipidomics were employed to study the effects of ANGPTL3 KD in IHH [60]. ANGPTL3 depletion led to statistically significant changes in several pathways related to lipid metabolism, such as the KEGG pathways 'Fatty acid 
metabolism', 'Biosynthesis of unsaturated fatty acids', 'Fatty acid elongation', 'Fatty acid degradation', 'Fatty acid biosynthesis', and 'Arachidonic acid metabolism'. These observations were accompanied by significant alterations in the total FA profile of the cells, showing a reduction of monounsaturated FA (MUFA) and an increase in both n-6 and n-3 PUFA. The relative MUFA depletion and PUFA increase were reflected in the lipid species profiles of major membrane phospholipids, the total levels of which, however, were not affected by the ANGPTL3 KD. In addition, there were similar FA changes observed in the species profile of CE, and the total CE level was reduced upon ANGPTL3 depletion. Interestingly, $\mathrm{Xu}$ et al. [32] found that in ANGPTL3 KO human hepatocellular carcinoma cells, depletion of ANGPTL3 resulted in intracellular TG and cholesterol accumulation as well as an increase of long-chain TG, TG 58:8 being the single most affected TG species. Inversely, short-chain TG and diacylglycerols decreased in the ANGPTL3 depleted cells.

Both n-3 and n-6 PUFA are precursors of bioactive lipid mediators, important signaling molecules orchestrating inflammation and its resolution. The imbalance of proresolving and pro-inflammatory lipid mediators plays a role in the development of atherosclerotic CVD [73-77]. ANGPTL3 KD resulted in our IHH model in an enhanced production of lipid mediators [60], likely as a consequence of both increased substrate PUFA availability and upregulation of mRNA expression of cytosolic phospholipase A2, which releases PUFA from membrane phospholipids [78,79]. Most of the mediators elevated in the ANGPTL3 KD hepatocytes have known functions in promoting the resolution of inflammation, recovery from cardiovascular events, protection from ER stress, or attenuation of insulin resistance and hepatic fat accumulation [80-85]. However, since also the production of several 20:4n-6 derived pro-inflammatory lipid mediators was enhanced in the ANGPTL3 KD cells, one should interpret the results with caution. Concerning the main topic of this review, the changes observed in the FA composition of hepatocellular lipids as well as the alterations of PUFA-derived lipid mediators are no doubt due to cell-autonomous effects of ANGPTL3 KD. They can be mediated through altered expression or activity of lipid-modifying enzymatic machineries, and possibly the apparatus responsible for the uptake and metabolism of lipids, FA and glucose from the growth medium.

\section{What does the plasma metabolome of ANGPTL3-deficient human subjects tell about their hepatic metabolism?}


Human plasma metabolome analysis revealed that ANGPTL3 LOF variant carriers have significantly higher concentrations of $3 \beta$-hydroxybutyrate (a ketone body) compared to normal human subjects, which may reflect enhanced hepatic $\beta$-oxidation of FA in the LOF variant carriers [86]. This could obviously result in reduced TG synthesis and packaging into newly synthesized VLDL particles, and thus be one of the mechanisms contributing to the low plasma apoB-100 lipoprotein levels in ANGPTL3-deficient or -depleted subjects. Of note, Wang et al. [31] found no evidence for enhanced hepatic FA oxidation in mice treated with Angptl3-inactivating antibody, consistent with the view that intra-hepatocellular KD of the gene may be required for such an effect. Similar to $\beta$-hydroxybutyrate, lactate was consistently higher at fasting and after a meal challenge in human subjects with complete ANGPTL3 deficiency [86]. This may indicate an enhanced conversion of pyruvate to lactate instead of its routing to the acetate pathway, consistent with the reduced concentration of acetate observed in the LOF carriers. These observations suggest that ANGPTL3 deficiency causes a modest but significant shift in the hepatic energy substrate utilization.

\section{ANGPTL3 and insulin sensitivity}

ANGPTL3 LOF carriers exhibit enhanced insulin sensitivity and low plasma free FA levels [16]. The decrease in free FAs observed in these subjects could be the result of reduced adipocyte lipolysis in the absence of ANGPTL3 [62]. Moreover, there is evidence for a regulatory link between insulin and ANGPTL3: Plasma ANGPTL3 was reduced upon insulin infusion in human subjects, and insulin treatment suppressed the expression and secretion of ANGPTL3 by $\mathrm{IHH}$ [6]. A recent study utilizing non-human primates shows that the plasma concentration of ANGPTL3 was elevated in fructose induced insulin resistance and correlated positively with HOMA-IR, an index of insulin resistance [87]. In an elegant study on Angpt/3 $/$ mice, Wang et al. [33] demonstrated an increase of insulin sensitivity, and reduced FA but clearly elevated glucose uptake into WAT in the fed state. This alteration in WAT substrate utilization could provide an explanation for the improvement of insulin sensitivity observed in both humans and mice deficient in ANGPTL3. A consistent effect of Angplt3 inactivation on insulin sensitivity in mice treated with ASO was reported by Graham et al. [23]. Ruhanen et al. [60] showed that PUFA-containing lipid species were enriched in ANGPTL3 KD hepatocytes and that a similar PUFA enrichment was present in the TG of lipoproteins derived from human ANGPTL3 LOF homozygote carriers. The impact of this 
altered lipid composition of the lipoproteins in the ANGPTL3 deficient subjects remains an intriguing topic of future study. However, it might affect the quality of FAs stored in adipocytes but also in tissues involved in ectopic lipid storage, promoting insulin sensitivity. To conclude, the mechanisms through which depletion of ANGPTL3 improves whole body insulin sensitivity are thus far not well understood. However, present evidence suggests that reduced FA uptake into WAT and lipolysis, as well as elevated glucose uptake into WAT may play roles here. Further, the enrichment of circulating lipoproteins with PUFA in subjects with low ANGPTL3 activity could favorably modify the fatty acid composition of tissues crucial for insulin sensitivity. Moreover, ANGPTL3 KD was reported to reduce gluconeogenic gene expression and reduce the secretion of TG-rich VLDL by hepatocytes in culture [30], with putative insulin sensitizing effects.

\section{Which physiological functions of ANGPTL3 can be considered cell autonomous?}

A number of in vitro findings made by employing cultured hepatocyte models suggest cellautonomous functions of ANGPTL3 in controlling hepatocellular VLDL secretion, $\beta$ lipoprotein uptake [32], or the insulin regulation of VLDL assembly/secretion [30]. Since functional impact in cultured hepatocytes cannot be attributed to alterations of LPL activity and the resulting FA fluxes, the above in vitro observations almost certainly reflect cellautonomous functions of the protein. The same holds true for the changes observed in the FA composition of lipids, and the alterations in the transcriptome of cultured $\mathrm{IHH}$ subjected to ANGPTL3 KD [60]. We are tempted to speculate that the alterations in gene expression observed in the hepatocytes depleted of ANGPTL3 could relate to the altered FA metabolism, considering that FAs and their derivatives regulate a number of key nuclear receptors or transcription factors, such as peroxisome proliferator activated receptors (PPAR), hepatic nuclear factors (HNF), retinoid $X$ receptors (RXR), and sterol regulatory element binding protein 1c (SREBP-1c) [88-91].

In vivo observations are more difficult to interpret from the point-of-view of cell autonomy, due to the complexity of biological systems. Most of the effects of ANGPTL3 antibody inhibition observed in mouse and man $[19,21,22,24,31,42]$ can be attributed to the activation of LPL and resulting alterations in FA fluxes between lipoproteins and tissues, as well as the resulting indirect effects on hepatic lipoprotein production. However, the in vivo plus in vitro ANGPTL3 KD study of Xu et al. [32] contains truly interesting evidence for a role of ANGPTL3 in controlling hepatic lipid metabolism cell-autonomously, demonstrating an 
enhancement of hepatic LDL/VLDL uptake, and provides evidence suggesting that this is due to elevated expression of LDLR and LRP1 by ANGPTL3-depleted hepatocytes (Fig. 3). Of note, significant reduction of LDL-C is observed not only in human subjects treated with ANGPTL3 ASO [23] but also upon Evinacumab treatment $[21,22,24]$. This suggests that an intrahepatocellular LDLR/LRP1 induction cannot be the major mechanism underlying the LDL-C lowering effect. It rather implies that altered fatty acid fluxes modulating hepatic VLDL assembly/secretion and the turn-over properties of the resulting $\beta$-lipoproteins must play a more important role.

One may wonder by what mechanism ANGPTL3, which has an N-terminal signal sequence, is synthesized through the endoplasmic reticulum (ER) membrane into the ER lumen and is secreted by hepatocytes through the canonical secretory route, could execute intracellular or cell-autonomous functions. Obviously, it could participate in lipoprotein assembly within the secretory pathway luminal compartments, in the ER, the Golgi apparatus and within secretory vesicles (Fig. 3). This type of intracellular function has in fact been demonstrated for another crucial regulator of lipoprotein metabolism, the phospholipid transfer protein (PLTP), a secreted plasma factor that also plays a major role in the assembly of apoB-containing lipoproteins in hepatocytes [92,93]. The intracellular function of PLTP most likely involves transfer of phospholipids to the nascent apoB lipoproteins within the secretory pathway. However, PLTP has also been found within the nucleus of neuronal cells, where it is suggested to execute as yet poorly understood functions [94]. Similar to PLTP, ANGPTL3 could regulate VLDL assembly and secretion through an activity within the secretory pathway of hepatocytes, where it is activated by furin cleavage [95]. Whether an isoform of ANGPTL3 could be found localized to the cytoplasmic or nuclear compartments is thus far not known. In the NCBI database there are no alternatively spliced variants of ANGPTL3 that would lack a signal sequence - however, this does not exclude their existence, or the signal sequence could under some circumstances be masked. Moreover, the protein secreted to the cell surface could be internalized through the endocytic pathway (Fig. 3) and execute activity within the endo-lysosomal compartments, recycle to the trans-Golgi, or possibly even exit the endomembrane system. These intriguing questions warrant detailed study in the future.

An interesting finding published almost 20 years ago showed that ANGPTL3 is able to bind integrin, a cell adhesion molecule [96]. The authors demonstrated in their cell- 
based assays with recombinant proteins and direct binding experiments that ANGPTL3 bound to $\alpha v \beta_{3}$-integrin and was thereby able to induce endothelial cell migration and adhesion. The Fibrinogen-like domain (FLD) of ANGPTL3 was sufficient for the integrin binding and induction of angiogenesis. The role of integrin $\alpha \vee \beta_{3}$ as a receptor for ANGPTL3 has been afterwards verified in other locations such as in kidney podocytes [97,98]. Interestingly, ANGPTL3 was in podocytes shown to induce actin filament rearrangement, mediated by integrin $\alpha_{v} \beta_{3}$-dependent FAK and PI3K phosphorylation and Rac1 activation. Actin cytoskeleton could also be a target of ANGPTL3-integrin action in the liver. Furthermore, the PI3K-Akt pathway is a crucial part of the signaling that controls TG metabolism in hepatocytes. We therefore speculate that hepatic ANGPTL3 deficiency could suppress integrin $\alpha v \beta_{3} / \mathrm{Pl} / 3 \mathrm{~K} / \mathrm{Akt}$ signaling and thereby affect liver TG homeostasis. ANGPTL3 secreted by hepatocytes could engage $\alpha v \beta_{3}$ on the surface of the same or nearby cells, resulting in cell-autonomous regulation of TG metabolism and VLDL secretion (Fig. 3). Moreover, ANGPTL3 may represent a local liver-specific angiogenic factor regulating vascular responses by binding to $\alpha v \beta_{3}$-integrin expressed on the liver endothelium.

To conclude, accumulating evidence suggests that manipulating the expression of hepatic ANGPTL3 does have cell-autonomous impacts on hepatocellular gene expression and lipid metabolism. The topic has thus far been addressed only in a handful of studies and definitely deserves more thorough investigation.

\section{Conclusions and future perspectives}

ANGPTL3 is a highly promising new cardiometabolic therapy target. Nucleic acid-based therapeutic approaches that dampen the hepatic expression of this protein not only affect its lipase inhibitor activity in circulation but also its putative, emerging intra-hepatocellular or hepatocyte cell-autonomous activities. The evidence thus far suggests that suppression of ANGPTL3 expression has quite significant effects on the hepatocyte transcriptome [60], glucose uptake, insulin sensitivity [23,30], LDL/VLDL uptake [32], VLDL assembly/secretion [23,32], the PUFA content of hepatocyte lipids, and the concentrations of PUFA-derived lipid mediators [60]. While most of these putatively cell-autonomous impacts of ANGPTL3 manipulation may be beneficial in nature and contribute to the cardioprotective capacity of 
ANGPTL3 suppression, it is important to realize that among them there may also be ones with potential to be harmful in long term. Therefore, detailed study of the intra-hepatocellular functions of ANGPTL3 is definitely warranted.

\section{Acknowledgments}

We thank Mikko Olkkonen for preparing the figures.

\section{Funding}

The work in the authors' group is supported by the Novo Nordisk Foundation (VMO), the Paavo Nurmi Foundation (VMO), the Liv och Hälsa Foundation (VMO), the Finnish Foundation for Cardiovascular Research (MJ, VMO), the Jane and Aatos Erkko Foundation (MJ), and the Magnus Ehrnrooth Foundation (ML, VMO).

\section{Conflicts of interest}

None.

\section{References}

[1] Kersten S. New insights into angiopoietin-like proteins in lipid metabolism and cardiovascular disease risk. Curr Opin Lipidol 30 (2019) 205-11.

[2] Conklin D, Gilbertson D, Taft DW, Maurer MF, Whitmore TE, Smith DL, et al. Identification of a mammalian angiopoietin-related protein expressed specifically in liver. Genomics 62 (1999) 477-82.

[3] Kaplan R, Zhang T, Hernandez M, Gan FX, Wright SD, Waters MG, et al. Regulation of the angiopoietin-like protein 3 gene by LXR. J Lipid Res 44 (2003) 136-43.

[4] Shimamura M, Matsuda M, Ando Y, Koishi R, Yasumo H, Furukawa H, et al. Leptin and insulin down-regulate angiopoietin-like protein 3 , a plasma triglyceride-increasing factor. Biochem Biophys Res Commun 322 (2004) 1080-5. 
[5] Fugier C, Tousaint JJ, Prieur X, Plateroti M, Samarut J, Delerive P. The lipoprotein lipase inhibitor ANGPTL3 is negatively regulated by thyroid hormone. J Biol Chem 281 (2006) 11553-9.

[6] Nidhina Haridas PA, Soronen J, Sädevirta S, Mysore R, Quagliarini F, Pasternack A, et al. Regulation of Angiopoietin-Like Proteins (ANGPTLs) 3 and 8 by Insulin. J Clin Endocrinol Metab 100 (2015) E1299-307.

[7] Quagliarini F, Wang Y, Kozlitina J, Grishin NV, Hyde R, Boerwinkle E, et al. Atypical angiopoietin-like protein that regulates ANGPTL3. Proc Natl Acad Sci U S A 109 (2012) 19751-6.

[8] Chi X, Britt EC, Shows HW, Hjelmaas AJ, Shetty SK, Cushing EM, et al. ANGPTL8 promotes the ability of ANGPTL3 to bind and inhibit lipoprotein lipase. Mol Metab 6 (2017) 1137-49.

[9] Kersten S, Mandard S, Tan NS, Escher P, Metzger D, Chambon P, et al. Characterization of the fasting-induced adipose factor FIAF, a novel peroxisome proliferator-activated receptor target gene. J Biol Chem 275 (2000) 28488-93.

[10] Romeo S, Yin W, Kozlitina J, Pennacchio LA, Boerwinkle E, Hobbs HH, et al. Rare loss-of-function mutations in ANGPTL family members contribute to plasma triglyceride levels in humans. J Clin Invest 119 (2009) 70-9.

[11] Koishi R, Ando Y, Ono M, Shimamura M, Yasumo H, Fujiwara T, et al. Angptl3 regulates lipid metabolism in mice. Nat Genet 30 (2002) 151-7.

[12] Köster A, Chao YB, Mosior M, Ford A, Gonzalez-DeWhitt PA, Hale JE, et al. Transgenic angiopoietin-like (angptl)4 overexpression and targeted disruption of angpt/4 and angpt|3: regulation of triglyceride metabolism. Endocrinology 146 (2005) 4943-50.

[13] Willer CJ, Sanna S, Jackson AU, Scuteri A, Bonnycastle LL, Clarke R, et al. Newly identified loci that influence lipid concentrations and risk of coronary artery disease. Nat Genet 40 (2008) 161-9.

[14] Musunuru K, Pirruccello JP, Do R, Peloso GM, Guiducci C, Sougnez C, et al. Exome sequencing, ANGPTL3 mutations, and familial combined hypolipidemia. N Engl J Med 363 (2010) 2220-7. 
[15] Minicocci I, Montali A, Robciuc MR, Quagliarini F, Censi V, Labbadia G, et al. Mutations in the ANGPTL3 gene and familial combined hypolipidemia: a clinical and biochemical characterization. J Clin Endocrinol Metab 97 (2012) E1266-75.

[16] Robciuc MR, Maranghi M, Lahikainen A, Rader D, Bensadoun A, Öörni K, et al. Angptl3 deficiency is associated with increased insulin sensitivity, lipoprotein lipase activity, and decreased serum free fatty acids. Arterioscler Thromb Vasc Biol 33 (2013) 1706-13.

[17] Shimizugawa T, Ono M, Shimamura M, Yoshida K, Ando Y, Koishi R, et al. ANGPTL3 decreases very low density lipoprotein triglyceride clearance by inhibition of lipoprotein lipase. J Biol Chem 277 (2002) 33742-8.

[18] Shimamura M, Matsuda M, Yasumo H, Okazaki M, Fujimoto K, Kono K, et al. Angiopoietin-like protein3 regulates plasma HDL cholesterol through suppression of endothelial lipase. Arterioscler Thromb Vasc Biol 27 (2007) 366-72.

[19] Gusarova V, Alexa CA, Wang Y, Rafique A, Kim JH, Buckler D, et al. ANGPTL3 blockade with a human monoclonal antibody reduces plasma lipids in dyslipidemic mice and monkeys. J Lipid Res 56 (2015) 1308-17.

[20] Stitziel NO, Khera AV, Wang X, Bierhals AJ, Vourakis AC, Sperry AE, et al. ANGPTL3 Deficiency and Protection Against Coronary Artery Disease. J Am Coll Cardiol 69 (2017) 2054-63.

[21] Dewey FE, Gusarova V, Dunbar RL, O'Dushlaine C, Schurmann C, Gottesman O, et al. Genetic and Pharmacologic Inactivation of ANGPTL3 and Cardiovascular Disease. N Engl J Med 377 (2017) 211-21.

[22] Gaudet D, Gipe DA, Pordy R, Ahmad Z, Cuchel M, Shah PK, et al. ANGPTL3 Inhibition in Homozygous Familial Hypercholesterolemia. N Engl J Med 377 (2017) 296-7.

[23] Graham MJ, Lee RG, Brandt TA, Tai LJ, Fu W, Peralta R, et al. Cardiovascular and Metabolic Effects of ANGPTL3 Antisense Oligonucleotides. N Engl J Med 377 (2017) 22232.

[24] Ahmad Z, Banerjee P, Hamon S, Chan KC, Bouzelmat A, Sasiela WJ, et al. Inhibition of Angiopoietin-Like Protein 3 With a Monoclonal Antibody Reduces Triglycerides in Hypertriglyceridemia. Circulation 140 (2019) 470-86.

[25] Hegele RA, Tsimikas S. Lipid-Lowering Agents. Circ Res 124 (2019) 386-404. 
[26] Lang W, Frishman WH. Angiopoietin-Like 3 Protein Inhibition: A New Frontier in LipidLowering Treatment. Cardiol Rev 27 (2019) 211-7.

[27] Wang X, Musunuru K. Angiopoietin-Like 3: From Discovery to Therapeutic Gene Editing. JACC Basic TransI Sci 4 (2019) 755-62.

[28] Arca M, D'Erasmo L, Minicocci I. Familial combined hypolipidemia: angiopoietin-like protein-3 deficiency. Curr Opin Lipidol 31 (2020) 41-8.

[29] Li J, Li L, Guo D, Li S, Zeng Y, Liu C, et al. Triglyceride metabolism and angiopoietinlike proteins in lipoprotein lipase regulation. Clin Chim Acta 503 (2020) 19-34.

[30] Tikka A, Soronen J, Laurila PP, Metso J, Ehnholm C, Jauhiainen M. Silencing of ANGPTL 3 (angiopoietin-like protein 3) in human hepatocytes results in decreased expression of gluconeogenic genes and reduced triacylglycerol-rich VLDL secretion upon insulin stimulation. Biosci Rep 34 (2014) e00160.

[31] Wang Y, Gusarova V, Banfi S, Gromada J, Cohen JC, Hobbs HH. Inactivation of ANGPTL3 reduces hepatic VLDL-triglyceride secretion. J Lipid Res 56 (2015) 1296-307.

[32] Xu YX, Redon V, Yu H, Querbes W, Pirruccello J, Liebow A, et al. Role of angiopoietin-like 3 (ANGPTL3) in regulating plasma level of low-density lipoprotein cholesterol. Atherosclerosis 268 (2018) 196-206.

[33] Wang Y, McNutt MC, Banfi S, Levin MG, Holland WL, Gusarova V, et al. Hepatic ANGPTL3 regulates adipose tissue energy homeostasis. Proc Natl Acad Sci U S A 112 (2015) 11630-5.

[34] Martin-Campos JM, Roig R, Mayoral C, Martinez S, Marti G, Arroyo JA, et al. Identification of a novel mutation in the ANGPTL3 gene in two families diagnosed of familial hypobetalipoproteinemia without APOB mutation. Clin Chim Acta 413 (2012) 5525.

[35] Noto D, Cefalu AB, Valenti V, Fayer F, Pinotti E, Ditta M, et al. Prevalence of ANGPTL3 and APOB gene mutations in subjects with combined hypolipidemia. Arterioscler Thromb Vasc Biol 32 (2012) 805-9.

[36] Pisciotta L, Favari E, Magnolo L, Simonelli S, Adorni MP, Sallo R, et al. Characterization of three kindreds with familial combined hypolipidemia caused by loss-offunction mutations of ANGPTL3. Circ Cardiovasc Genet 5 (2012) 42-50. 
[37] Fazio S, Sidoli A, Vivenzio A, Maietta A, Giampaoli S, Menotti A, et al. A form of familial hypobetalipoproteinaemia not due to a mutation in the apolipoprotein $B$ gene. $J$ Intern Med 229 (1991) 41-7.

[38] Lotta LA, Stewart ID, Sharp SJ, Day FR, Burgess S, Luan J, et al. Association of Genetically Enhanced Lipoprotein Lipase-Mediated Lipolysis and Low-Density Lipoprotein Cholesterol-Lowering Alleles With Risk of Coronary Disease and Type 2 Diabetes. JAMA Cardiol 3 (2018) 957-66.

[39] Chadwick AC, Evitt NH, Lv W, Musunuru K. Reduced Blood Lipid Levels With In Vivo CRISPR-Cas9 Base Editing of ANGPTL3. Circulation 137 (2018) 975-7.

[40] Crooke ST, Baker BF, Witztum JL, Kwoh TJ, Pham NC, Salgado N, et al. The Effects of 2'-O-Methoxyethyl Containing Antisense Oligonucleotides on Platelets in Human Clinical Trials. Nucleic Acid Ther 27 (2017) 121-9.

[41] Frazier KS. Antisense oligonucleotide therapies: the promise and the challenges from a toxicologic pathologist's perspective. Toxicol Pathol 43 (2015) 78-89.

[42] Pouwer MG, Pieterman EJ, Worms N, Keijzer N, Jukema JW, Gromada J, et al. Alirocumab, evinacumab, and atorvastatin triple therapy regresses plaque lesions and improves lesion composition in mice. J Lipid Res 61 (2020) 365-75.

[43] Ference BA, Kastelein JJP, Ray KK, Ginsberg HN, Chapman MJ, Packard CJ, et al. Association of Triglyceride-Lowering LPL Variants and LDL-C-Lowering LDLR Variants With Risk of Coronary Heart Disease. JAMA 321 (2019) 364-73.

[44] Minicocci I, Santini S, Cantisani V, Stitziel N, Kathiresan S, Arroyo JA, et al. Clinical characteristics and plasma lipids in subjects with familial combined hypolipidemia: a pooled analysis. J Lipid Res 54 (2013) 3481-90.

[45] Young SG. Recent progress in understanding apolipoprotein B. Circulation 82 (1990) 1574-94.

[46] Linton MF, Farese RV,Jr, Young SG. Familial hypobetalipoproteinemia. J Lipid Res 34 (1993) 521-41.

[47] Schonfeld G, Patterson BW, Yablonskiy DA, Tanoli TS, Averna M, Elias N, et al. Fatty liver in familial hypobetalipoproteinemia: triglyceride assembly into VLDL particles is affected by the extent of hepatic steatosis. J Lipid Res 44 (2003) 470-8. 
[48] Tanoli T, Yue P, Yablonskiy D, Schonfeld G. Fatty liver in familial hypobetalipoproteinemia: roles of the APOB defects, intra-abdominal adipose tissue, and insulin sensitivity. J Lipid Res 45 (2004) 941-7.

[49] Mouzaki M, Shah A, Arce-Clachar AC, Hardy J, Bramlage K, Xanthakos SA. Extremely low levels of low-density lipoprotein potentially suggestive of familial hypobetalipoproteinemia: A separate phenotype of NAFLD? J Clin Lipidol 13 (2019) 42531.

[50] Hooper AJ, Heeks L, Robertson K, Champain D, Hua J, Song S, et al. Lipoprotein Metabolism in APOB L343V Familial Hypobetalipoproteinemia. J Clin Endocrinol Metab 100 (2015) E1484-90.

[51] Hooper AJ, Robertson K, Champain D, Hua J, Song S, Parhofer KG, et al. Lipoprotein metabolism in an apoB-80 familial hypobetalipoproteinemia heterozygote. Clin Biochem 49 (2016) 720-2.

[52] Di Costanzo A, Di Leo E, Noto D, Cefalu AB, Minicocci I, Polito L, et al. Clinical and biochemical characteristics of individuals with low cholesterol syndromes: A comparison between familial hypobetalipoproteinemia and familial combined hypolipidemia. J Clin Lipidol 11 (2017) 1234-42.

[53] Yilmaz Y, Ulukaya E, Atug O, Dolar E. Serum concentrations of human angiopoietinlike protein 3 in patients with nonalcoholic fatty liver disease: association with insulin resistance. Eur J Gastroenterol Hepatol 21 (2009) 1247-51.

[54] Morinaga J, Zhao J, Endo M, Kadomatsu T, Miyata K, Sugizaki T, et al. Association of circulating ANGPTL 3, 4, and 8 levels with medical status in a population undergoing routine medical checkups: A cross-sectional study. PLoS One 13 (2018) e0193731.

[55] Hess AL, Carayol J, Blaedel T, Hager J, Di Cara A, Astrup A, et al. Analysis of circulating angiopoietin-like protein 3 and genetic variants in lipid metabolism and liver health: the DiOGenes study. Genes Nutr 13 (2018) 7,018-0597-3. eCollection 2018.

[56] Diab DL, Yerian L, Schauer P, Kashyap SR, Lopez R, Hazen SL, et al. Cytokeratin 18 fragment levels as a noninvasive biomarker for nonalcoholic steatohepatitis in bariatric surgery patients. Clin Gastroenterol Hepatol 6 (2008) 1249-54.

[57] Szalowska E, Dijkstra M, Elferink MG, Weening D, de Vries M, Bruinenberg M, et al. Comparative analysis of the human hepatic and adipose tissue transcriptomes during 
LPS-induced inflammation leads to the identification of differential biological pathways and candidate biomarkers. BMC Med Genomics 4 (2011) 71,8794-4-71.

[58] Liu X, Men P, Wang Y, Zhai S, Zhao Z, Liu G. Efficacy and Safety of Lomitapide in Hypercholesterolemia. Am J Cardiovasc Drugs 17 (2017) 299-309.

[59] Parham JS, Goldberg AC. Mipomersen and its use in familial hypercholesterolemia. Expert Opin Pharmacother 20 (2019) 127-31.

[60] Ruhanen H, Haridas PAN, Minicocci I, Taskinen JH, Palmas F, di Costanzo A, et al. ANGPTL3 deficiency alters the lipid profile and metabolism of cultured hepatocytes and human lipoproteins. Biochim Biophys Acta Mol Cell Biol Lipids 1865 (2020) 158679.

[61] Armstrong SM, Sugiyama MG, Fung KY, Gao Y, Wang C, Levy AS, et al. A novel assay uncovers an unexpected role for SR-BI in LDL transcytosis. Cardiovasc Res 108 (2015) 268-77.

[62] Shimamura M, Matsuda M, Kobayashi S, Ando Y, Ono M, Koishi R, et al. Angiopoietin-like protein 3 , a hepatic secretory factor, activates lipolysis in adipocytes. Biochem Biophys Res Commun 301 (2003) 604-9.

[63] Burnett JR, Wilcox LJ, Telford DE, Kleinstiver SJ, Barrett PH, Newton RS, et al. Inhibition of ACAT by avasimibe decreases both VLDL and LDL apolipoprotein B production in miniature pigs. J Lipid Res 40 (1999) 1317-27.

[64] Cianflone KM, Yasruel Z, Rodriguez MA, Vas D, Sniderman AD. Regulation of apoB secretion from HepG2 cells: evidence for a critical role for cholesteryl ester synthesis in the response to a fatty acid challenge. J Lipid Res 31 (1990) 2045-55.

[65] Mason TM. The role of factors that regulate the synthesis and secretion of very-lowdensity lipoprotein by hepatocytes. Crit Rev Clin Lab Sci 35 (1998) 461-87.

[66] Ooi EM, Watts GF, Ng TW, Barrett PH. Effect of dietary Fatty acids on human lipoprotein metabolism: a comprehensive update. Nutrients 7 (2015) 4416-25.

[67] Rong X, Wang B, Dunham MM, Hedde PN, Wong JS, Gratton E, et al. Lpcat3dependent production of arachidonoyl phospholipids is a key determinant of triglyceride secretion. Elife 4 (2015) 10.7554/eLife.06557.

[68] Rong Guo X, Li Wang X, Chen Y, Hong Yuan Y, Mei Chen Y, Ding Y, et al. ANGPTL8/betatrophin alleviates insulin resistance via the Akt-GSK3beta or Akt-FoxO1 pathway in HepG2 cells. Exp Cell Res 345 (2016) 158-67. 
[69] Zhang Y, Guo X, Yan W, Chen Y, Ke M, Cheng C, et al. ANGPTL8 negatively regulates NF-kappaB activation by facilitating selective autophagic degradation of IKKgamma. Nat Commun 8 (2017) 2164,017-02355-w.

[70] Kovrov O, Kristensen KK, Larsson E, Ploug M, Olivecrona G. On the mechanism of angiopoietin-like protein 8 for control of lipoprotein lipase activity. J Lipid Res 60 (2019) 783-93.

[71] Haller JF, Mintah IJ, Shihanian LM, Stevis P, Buckler D, Alexa-Braun CA, et al. ANGPTL8 requires ANGPTL3 to inhibit lipoprotein lipase and plasma triglyceride clearance. J Lipid Res 58 (2017) 1166-73.

[72] Wang Y, Quagliarini F, Gusarova V, Gromada J, Valenzuela DM, Cohen JC, et al. Mice lacking ANGPTL8 (Betatrophin) manifest disrupted triglyceride metabolism without impaired glucose homeostasis. Proc Natl Acad Sci U S A 110 (2013) 16109-14.

[73] Akagi D, Chen M, Toy R, Chatterjee A, Conte MS. Systemic delivery of proresolving lipid mediators resolvin D2 and maresin 1 attenuates intimal hyperplasia in mice. FASEB J 29 (2015) 2504-13.

[74] Fredman G, Hellmann J, Proto JD, Kuriakose G, Colas RA, Dorweiler B, et al. An imbalance between specialized pro-resolving lipid mediators and pro-inflammatory leukotrienes promotes instability of atherosclerotic plaques. Nat Commun 7 (2016) 12859.

[75] Pirault J, Bäck M. Lipoxin and Resolvin Receptors Transducing the Resolution of Inflammation in Cardiovascular Disease. Front Pharmacol 9 (2018) 1273.

[76] Bäck M, Yurdagul A,Jr, Tabas I, Öörni K, Kovanen PT. Inflammation and its resolution in atherosclerosis: mediators and therapeutic opportunities. Nat Rev Cardiol 16 (2019) 389-406.

[77] Gerlach BD, Marinello M, Heinz J, Rymut N, Sansbury BE, Riley CO, et al. Resolvin D1 promotes the targeting and clearance of necroptotic cells. Cell Death Differ (2019) .

[78] Shikano M, Masuzawa Y, Yazawa K, Takayama K, Kudo I, Inoue K. Complete discrimination of docosahexaenoate from arachidonate by $85 \mathrm{kDa}$ cytosolic phospholipase A2 during the hydrolysis of diacyl- and alkenylacylglycerophosphoethanolamine. Biochim Biophys Acta 1212 (1994) 211-6. 
[79] Batchu KC, Hänninen S, Jha SK, Jeltsch M, Somerharju P. Factors regulating the substrate specificity of cytosolic phospholipase A2-alpha in vitro. Biochim Biophys Acta 1861 (2016) 1597-604.

[80] Elajami TK, Colas RA, Dalli J, Chiang N, Serhan CN, Welty FK. Specialized proresolving lipid mediators in patients with coronary artery disease and their potential for clot remodeling. FASEB J 30 (2016) 2792-801.

[81] Kain V, Liu F, Kozlovskaya V, Ingle KA, Bolisetty S, Agarwal A, et al. Resolution Agonist 15-epi-Lipoxin A4 Programs Early Activation of Resolving Phase in PostMyocardial Infarction Healing. Sci Rep 7 (2017) 9999,017-10441-8.

[82] Rius B, Duran-Guell M, Flores-Costa R, Lopez-Vicario C, Lopategi A, Alcaraz-Quiles $J$, et al. The specialized proresolving lipid mediator maresin 1 protects hepatocytes from lipotoxic and hypoxia-induced endoplasmic reticulum stress. FASEB J 31 (2017) 5384-98.

[83] Jung TW, Kyung EJ, Kim HC, Shin YK, Lee SH, Park ES, et al. Protectin DX Ameliorates Hepatic Steatosis by Suppression of Endoplasmic Reticulum Stress via AMPK-Induced ORP150 Expression. J Pharmacol Exp Ther 365 (2018) 485-93.

[84] Dakin SG, Colas RA, Newton J, Gwilym S, Jones N, Reid HAB, et al. 15-Epi-LXA4 and MaR1 counter inflammation in stromal cells from patients with Achilles tendinopathy and rupture. FASEB J 33 (2019) 8043-54.

[85] Jung TW, Ahn SH, Shin JW, Kim HC, Park ES, Abd El-Aty AM, et al. Protectin DX ameliorates palmitate-induced hepatic insulin resistance through AMPK/SIRT1-mediated modulation of fetuin-A and SeP expression. Clin Exp Pharmacol Physiol 46 (2019) 898909 .

[86] Tikkanen E, Minicocci I, Hällfors J, Di Costanzo A, D'Erasmo L, Poggiogalle E, et al. Metabolomic Signature of Angiopoietin-Like Protein 3 Deficiency in Fasting and Postprandial State. Arterioscler Thromb Vasc Biol 39 (2019) 665-74.

[87] Butler AA, Graham JL, Stanhope KL, Wong S, King S, Bremer AA, et al. Role of angiopoietin-like protein 3 in sugar-induced dyslipidemia in rhesus macaques: suppression by fish oil or RNAi. J Lipid Res 61 (2020) 376-86.

[88] Jump DB, Botolin D, Wang Y, Xu J, Christian B, Demeure O. Fatty acid regulation of hepatic gene transcription. J Nutr 135 (2005) 2503-6. 
[89] Varga T, Czimmerer Z, Nagy L. PPARs are a unique set of fatty acid regulated transcription factors controlling both lipid metabolism and inflammation. Biochim Biophys Acta 1812 (2011) 1007-22.

[90] Pawlak M, Lefebvre P, Staels B. Molecular mechanism of PPARalpha action and its impact on lipid metabolism, inflammation and fibrosis in non-alcoholic fatty liver disease. $J$ Hepatol 62 (2015) 720-33.

[91] Kim KH, Moore DD. Regulation of Liver Energy Balance by the Nuclear Receptors Farnesoid X Receptor and Peroxisome Proliferator Activated Receptor alpha. Dig Dis 35 (2017) 203-9.

[92] Okazaki H, Goldstein JL, Brown MS, Liang G. LXR-SREBP-1c-phospholipid transfer protein axis controls very low density lipoprotein (VLDL) particle size. J Biol Chem 285 (2010) 6801-10.

[93] Manchekar M, Liu Y, Sun Z, Richardson PE, Dashti N. Phospholipid transfer protein plays a major role in the initiation of apolipoprotein B-containing lipoprotein assembly in mouse primary hepatocytes. J Biol Chem 290 (2015) 8196-205.

[94] Vuletic S, Dong W, Wolfbauer G, Day JR, Albers JJ. PLTP is present in the nucleus, and its nuclear export is CRM1-dependent. Biochim Biophys Acta 1793 (2009) 584-91.

[95] Essalmani R, Susan-Resiga D, Chamberland A, Asselin MC, Canuel M, Constam D, et al. Furin is the primary in vivo convertase of angiopoietin-like 3 and endothelial lipase in hepatocytes. J Biol Chem 288 (2013) 26410-8.

[96] Camenisch G, Pisabarro MT, Sherman D, Kowalski J, Nagel M, Hass P, et al. ANGPTL3 stimulates endothelial cell adhesion and migration via integrin alpha vbeta 3 and induces blood vessel formation in vivo. J Biol Chem 277 (2002) 17281-90.

[97] Liu J, Gao X, Zhai Y, Shen Q, Sun L, Feng C, et al. A novel role of angiopoietin-like-3 associated with podocyte injury. Pediatr Res 77 (2015) 732-9.

[98] Dai R, Lin Y, Liu H, Rao J, Zhai Y, Zha X, et al. A vital role for Angptl3 in the PANinduced podocyte loss by affecting detachment and apoptosis in vitro. BMC Nephrol 16 (2015) 38,015-0034-4.

[99] Biterova E, Esmaeeli M, Alanen HI, Saaranen M, Ruddock LW. Structures of Angptl3 and Angptl4, modulators of triglyceride levels and coronary artery disease. Sci Rep 8 (2018) 6752,018-25237-7. 


\section{Figure captions}

Fig. 1. The structure of ANGPTL3 and its functional partner ANGPTL8. Abbreviations: SS, signal sequence (translocation into the ER for secretion); SE1, LPL-specific epitope; CCD, coiled-coil domain; LR, linker region; FLD, fibrinogen-like domain; LPL, lipoprotein lipase. The SE1 epitope is required for binding LPL and inhibiting its activity. The coiled-coil domains mediate oligomerization of ANGPTL3 and -8 with each other and possibly with other partners. The FLD (absent in ANGPTL8) mediates binding of ANGPTL3 to its cellular receptor $\alpha \vee \beta_{3}$-integrin, mediating signaling responses and the function of ANGPTL3 in angiogenesis. The image of ANGPTL3 FLD structure was reproduced from [99] under a Creative Commons license http://creativecommons.org/licenses/by/4.0/.

Fig. 2. The impacts of hepatic loss of ANGPTL3 expression or its antibody-mediated inhibition in circulation, on metabolic fluxes in white adipose tissue (WAT), striated muscle and liver. The image collates data from studies of human subjects with loss-of-function variants, Angpt/3-1- knock-out mice, pharmacologic inhibition studies in human or mice, and work carried out on cultured hepatocyte models. (A) Suppression of hepatocyte ANGPTL3 expression reduces the post-prandial routing of fatty acids (FA) from triglyceride-rich lipoproteins (TRL) for storage in WAT, while increasing glucose (Glc) uptake into WAT and adipose de novo lipogenesis (DNL). Meanwhile it increases the hepatocyte uptake of Glc, $L D L$, and TRL remnants (TRL remn.), and inhibits the secretion of VLDL by the liver. (B) Inhibition of circulating ANGPTL3 with a humanized antibody (Ab) enhances the activity of LPL on TRL at oxidative tissues such as muscle, esulting in elevated FA uptake into these tissues and reduced FA flux to the liver. The reduced FA availability in the liver in turn reduces the hepatic secretion of VLDL triglycerides. Of note, the loss or knock-down of ANGPTL3 in hepatocytes also results in the same metabolic effects as its inhibition in the circulation; The physiologic consequences of depleting ANGPTL3 expression thus represent a combination of the effects in (A) and (B). 
Fig. 3. The biosynthesis and putative cell autonomous functions of ANGPTL3 within hepatocytes. Points where ANGPTL3 can potentially execute cell autonomous functions are indicated with numerals (1-4). In the nucleus ANGPTL3 expression is regulated positively by liver $X$ receptors (LXR) and hepatic nuclear factor $1 \alpha(H N F 1 \alpha)$, and negatively by insulin, leptin, and thyroid hormone receptor $\beta$ (TRB). After synthesis and translocation into the endoplasmic reticulum, ANGPTL3 can form a complex with the related ANGPTL8, and the proteins are transported through the secretory pathway; Part of ANGPTL3 is on the way cleaved by the protease furin or other kexin-type proteases, which increases its biological activity. (1) Within the secretory pathway lumen ANGPTL3 may potentially regulate the assembly/lipidation/secretion of nascent VLDL particles. (2) After reaching the cell surface ANGPTL3 can via its fibrinogen-like domain bind to av $\beta_{3}$-integrin or other as yet unknown receptors to induce signaling responses within the cell. These include the PI3K/Akt/mTOR pathway, which plays an important role in controlling VLDL assembly and secretion. Moreover, this pathway regulates glucose (Glc) uptake and metabolism. (3) ANGPTL3 bound to cell surface receptors could also be internalized via endocytosis, and execute intracellular functions within endosomes, or be routed from these organelles to the Golgi complex. (4) Depletion of ANGPTL3 in hepatocytes is reported to enhance the expression of LDL-receptor (LDLR) and LDLR-related protein 1 (LRP1), and to increase the hepatocellular uptake of $L D L$ and TRL remnants (TRL remn). In addition to these four putative mechanisms, ANGPTL3 depletion is reported to increase the polyunsaturated fatty acid (PUFA) content of hepatocyte lipids, the synthesis of PUFA-derived lipid mediators, and possibly to stimulate the $\beta$-oxidation of fatty acids - processes which could reflect cell autonomous functions of the protein. 
Fig. 1
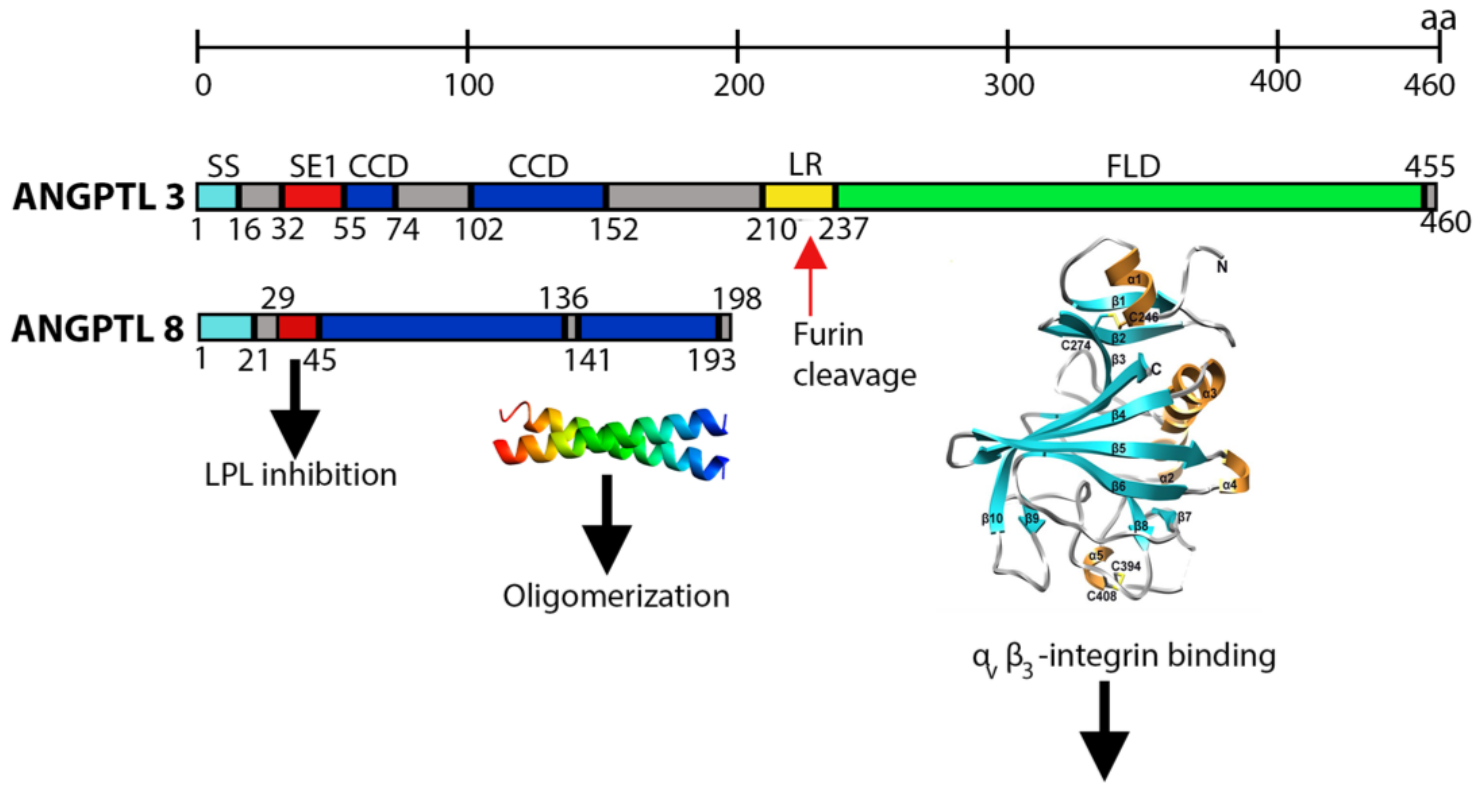

Signaling

Angiogenesis 
Fig. 2

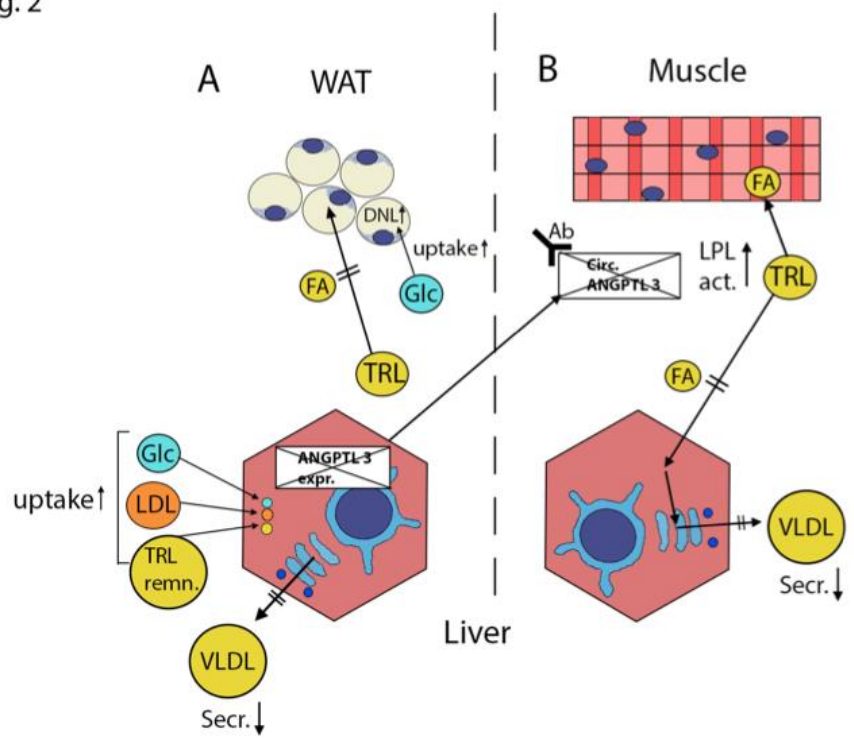


Fig. 3

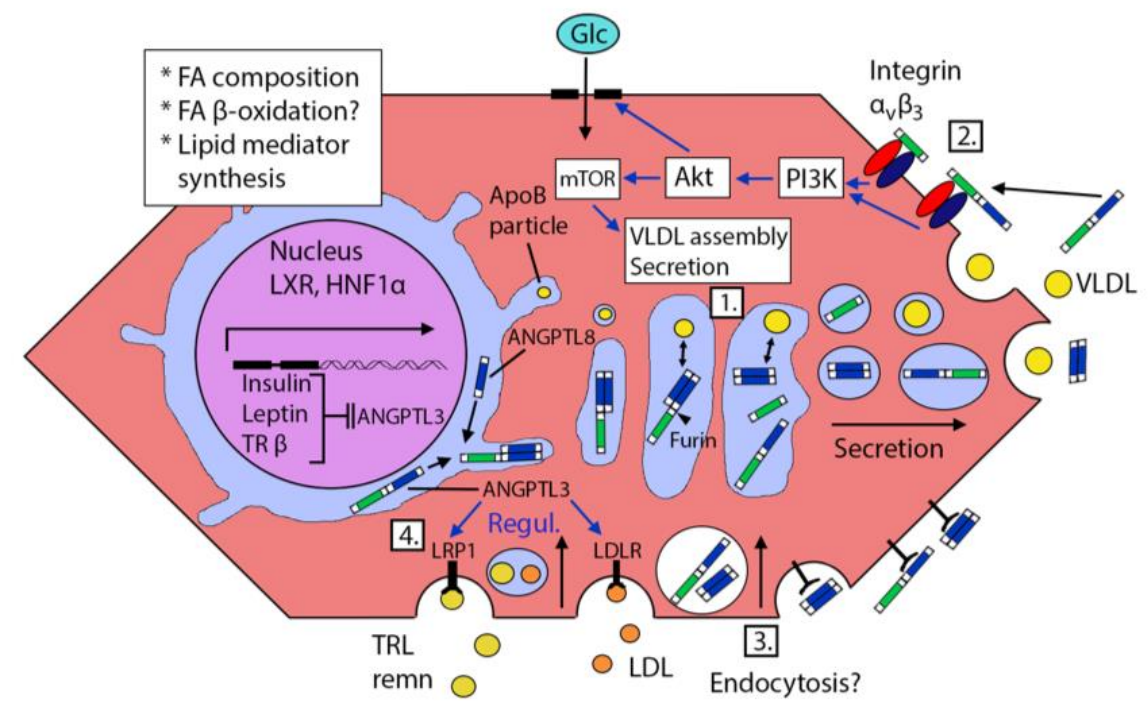

